\title{
Robustness of Sequential Probability Ratio Tests in Case of Nuisance Parameters
}

\author{
Karl-Heinz Eger ${ }^{1}$ and Evgeni Borisovich Tsoy ${ }^{2}$ \\ ${ }^{1}$ Chemnitz University of Technology, Department of Mathematics, Chemnitz, Germany \\ ${ }^{2}$ Novosibirsk State Technical University, Department of Applied Mathematics, Novosibirsk, Russia
}

\begin{abstract}
This paper deals with the computation of OCand ASN-function of sequential probability ratio tests in the multi-parameter case. In generalization of the method of conjugated parameter pairs Wald-like approximations are presented for the OC- and ASN-function. These characteristics can be used describing robustness properties of a sequential test in case of nuisance parameters. As examples tests are considered for the mean and the variance of a normal distribution.
\end{abstract}

\section{INTRODUCTION}

This paper deals with sequential probability ratio tests in the multi-parameter case in the following sense. Let $X_{1}, X_{2}, \ldots$ be a sequence of independent and identically distributed random variables with density function $f_{\vec{\theta}}(x)$ with respect to some measure $\mu$. Let $\vec{\theta}=\left(\theta_{1}, \theta_{2}\right)$ be a twodimensional parameter with values in a parameter space $\Theta$. Our aim is to discriminate between two simple onedimensional hypotheses, e.g. for the parameter $\theta_{1}$,

$$
H_{0}: \theta_{1}=\theta_{10} \text { against } H_{1}: \theta_{1}=\theta_{11},
$$

$\theta_{10} \neq \theta_{11}$, by means of a sequential probability ratio test based on the sequence $X_{1}, X_{2}, \ldots$.

We suppose that the second parameter $\theta_{2}$ is known and possesses the value $\theta_{2}=\theta_{20}$. In this context the parameter $\theta_{2}$ is a so-called nuisance or disturbing parameter. Special variants of such tests are e.g. tests for the mean of a normal distribution with known variance, for instance in case of testing the mean with known measuring accuracy, or tests for the variance of a normal distribution with known mean, respectively. Other examples in this sense are one-dimensional tests for parameters of Weibull or gamma distributions.

Then the question arises, what happens with the statistical properties of our test if the true value of the nuisance parameter $\theta_{2}$ is different from the assumed value $\theta_{20}$ or how robust is our test in view of modifications of true value of nuisance parameter.

This paper presents a method how we can describe the robustness of a sequential probability ratio test in case of nuisance parameters by means of generalized Wald approximations for the operating characteristic function (OC-function) and average sample number function (ASN-function). A helpful tool in this context is the socalled principle of conjugated parameter pairs. Especially we will consider examples for testing the mean or the variance of a normal distribution in case of nuisance parameters.

\section{The SEQUENTIAL PROBABILITY RATIO TEST}

We consider a sequential probability ratio test (SPRT) for discriminating between two simple two-dimensional hypotheses

$$
H_{0}: \theta_{1}=\theta_{10}, \theta_{2}=\theta_{20} \text { and } H_{1}: \theta_{1}=\theta_{11}, \theta_{2}=\theta_{21}
$$

with $\theta_{10} \neq \theta_{11}$ or/and $\theta_{21} \neq \theta_{20}$. This test is a generalization of the test for hypotheses (1) considered above. For instance, we get the hypotheses (1) if we put $\theta_{20}=\theta_{21}$.

Let $L_{n, \vec{\theta}_{0}, \vec{\theta}_{1}}$ be the likelihood ratio on stage $n, n=$ $1,2, \ldots$ then we have

$$
L_{n, \vec{\theta}_{0}, \vec{\theta}_{1}}=\prod_{i=1}^{n} \frac{f_{\vec{\theta}_{1}}\left(X_{i}\right)}{f_{\vec{\theta}_{0}}\left(X_{i}\right)} .
$$

To given stopping bounds $B$ and $A, 0<B<1<A<$ $\infty$, the sample size $N$ and the terminal decision rule $\delta$ of WALD's sequential probability ratio test are defined by

$$
N=\min \left\{n \geq 1: L_{n, \vec{\theta}_{0}, \vec{\theta}_{1}} \notin(B, A)\right\}
$$

and

$$
\delta=1_{\left\{L_{N, \vec{\theta}_{0}, \vec{\theta}_{1}} \leq B\right\}} .
$$

That means, we continue observations for $n=1,2, \ldots$ as long as the critical inequality $B<L_{n, \vec{\theta}_{0}, \vec{\theta}_{1}}<A$ holds. If

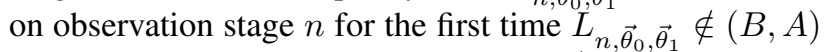
and if then $L_{n, \vec{\theta}_{0}, \vec{\theta}_{1}} \leq B$ or $L_{n, \vec{\theta}_{0}, \vec{\theta}_{1} \geq A \text { holds we accept }}$ the hypothesis $H_{0}$ or $H_{1}$, respectively. We denote this SPRT by $S(B, A)$.

The most important characteristics with respect to a description of the statistical properties of our test are the operating characteristic function (OC-function) $Q(\vec{\theta})=$ $E_{\vec{\theta}} \delta, \vec{\theta} \in \Theta$, and the average sample number function (ASN-function) $E_{\vec{\theta}} N, \vec{\theta} \in \Theta$.

If $P_{\vec{\theta}}\left(L_{1, \vec{\theta}_{0}, \vec{\theta}_{1}}=1\right)<1$ then we have $P_{\vec{\theta}}(N<\infty)=$ 1 and $E_{\vec{\theta}} N \stackrel{<}{<} \infty$. Moreover, the WALD-WolfowitzTheorem holds. That means, the test $S(B, A)$ minimizes the average sample number function for $\vec{\theta}=\vec{\theta}_{0}$ and $\vec{\theta}=\vec{\theta}_{1}$ among all tests whose error probabilities are not greater than the error probabilities of WALD's SPRT at $\vec{\theta}=\overrightarrow{\theta_{0}}$ and $\vec{\theta}=\overrightarrow{\theta_{1}}$.

The general problem of WALD's SPRT consists in the computation of its characteristics, e.g., the OC-function or the ASN-function. We will demonstrate that the so-called method of conjugate parameter pairs (see [3]) can be extended to the case considered here obtaining WALDlike approximations for the $\mathrm{OC}$ - and $\mathrm{ASN}$-function.

\section{A. The Wald approximations}

The OC- and ASN-function of test $S(B, A)$ can be computed approximately in sense of the so-called WALD approximations by means of conjugated parameter pairs as follows [3]. 
DEFINITION. Two parameter pairs $\left(\vec{\theta}, \overrightarrow{\theta^{\prime}}\right)$ and $\left(\vec{\theta}_{0}, \vec{\theta}_{1}\right) \in$ $\Theta \times \Theta$ are said to be conjugated, if a real number $h, h \neq 0$, exists, such that

$$
L_{n, \vec{\theta}, \vec{\theta}^{\prime}}=L_{n, \vec{\theta}_{0}, \vec{\theta}_{1}}^{h}, \quad n=1,2, \ldots,
$$

holds. We write: $\left(\vec{\theta}, \vec{\theta}^{\prime}\right) \stackrel{h}{\sim}\left(\vec{\theta}_{0}, \vec{\theta}_{1}\right)$.

If $\left(\vec{\theta}, \vec{\theta}^{\prime}\right) \stackrel{h}{\sim}\left(\vec{\theta}_{0}, \vec{\theta}_{1}\right)$ the OC-function $Q(\vec{\theta})$ and the power function $M(\vec{\theta})=E_{\vec{\theta}}(1-\delta), \vec{\theta} \in \Theta$, of test $S(B, A)$ satisfy the relations

$$
\frac{Q\left(\vec{\theta}^{\prime}\right)}{Q(\vec{\theta})}=E_{\vec{\theta}}\left(L_{N, \vec{\theta}_{0}, \vec{\theta}_{1}}^{h} \mid H_{0} \text { is acc. }\right) \leq B^{h}
$$

and

$$
\frac{M\left(\vec{\theta}^{\prime}\right)}{M(\vec{\theta})}=E_{\vec{\theta}}\left(L_{N, \vec{\theta}_{0}, \vec{\theta}_{1}}^{h} \mid H_{1} \text { is acc. }\right) \geq A^{h},
$$

where in case of

$$
\begin{aligned}
& P_{\vec{\theta}}\left(L_{N, \vec{\theta}_{0}, \vec{\theta}_{1}}=B \mid H_{0} \text { is accepted }\right) \\
& =P_{\vec{\theta}}\left(L_{N, \vec{\theta}_{0}, \vec{\theta}_{1}}=A \mid H_{1} \text { is accepted }\right)=1
\end{aligned}
$$

the equals signs hold. We remark, that in case of $P_{\vec{\theta}}(N<$ $\infty)=1$ (closed test) moreover $M(\vec{\theta})=1-Q(\vec{\theta})$ holds. A sufficient condition for closeness is, for instance, $P_{\vec{\theta}}\left(L_{1, \vec{\theta}_{0}, \vec{\theta}_{1}}=1\right)<1$ (see e.g. [3]).

\section{B. The OC-function}

For a closed test $S(B, A)$ we get in case of (5) by $\left(\vec{\theta}, \overrightarrow{\theta^{\prime}}\right) \stackrel{h}{\sim}\left(\vec{\theta}_{0}, \vec{\theta}_{1}\right),(3)$ and (4) for the OC-function

$$
Q(\vec{\theta})=Q^{*}(\vec{\theta})=\frac{A^{h}-1}{A^{h}-B^{h}}
$$

and

$$
Q\left(\vec{\theta}^{\prime}\right)=Q^{*}\left(\vec{\theta}^{\prime}\right)=B^{h} Q^{*}(\vec{\theta}) .
$$

If condition (5) holds approximately, that means the excess over the stopping boundaries is negligible when the test ends, e.g., $L_{n, \vec{\theta}_{0}, \vec{\theta}_{1}} \approx B$ or $L_{n, \vec{\theta}_{0}, \vec{\theta}_{1}} \approx A$ when $N=n$ then we have $Q(\vec{\theta}) \approx Q^{*}(\vec{\theta})$ and $Q\left(\vec{\theta}^{\prime}\right) \approx Q^{*}\left(\vec{\theta}^{\prime}\right)=$ $B^{h} Q^{*}(\vec{\theta})$. This are the famous WALD approximations for the OC-function. If to given $\vec{\theta}$ no $h \neq 0$ and no parameter vector $\vec{\theta}^{\prime} \neq \vec{\theta}$ exist such that $\left(\vec{\theta}, \vec{\theta}^{\prime}\right) \stackrel{h}{\sim}\left(\vec{\theta}_{0}, \vec{\theta}_{1}\right)$, e.g., in case of $E_{\vec{\theta}} Z_{1, \vec{\theta}_{0}, \vec{\theta}_{1}}=E_{\vec{\theta}} \ln L_{1, \vec{\theta}_{0}, \vec{\theta}_{1}}=0$, the WALD approximation for the OC-function can be extended by $Q(\vec{\theta}) \approx Q^{*}(\vec{\theta})=\ln A /(\ln A-\ln B)$ for $E_{\vec{\theta}} Z_{1, \vec{\theta}_{0}, \vec{\theta}_{1}}=0$.

\section{The stopping bounds}

Under condition (5) we obtain a test $S(B, A)$ at size $(\alpha, \beta), 0<\alpha, \beta<1, \alpha+\beta<1$, that means $Q\left(\vec{\theta}_{0}\right)=1-\alpha$ and $Q\left(\vec{\theta}_{1}\right)=\beta$, if the stopping boundaries $B$ and $A$ satisfy the conditions

$$
B=B^{*}=\frac{1-\beta}{\alpha} \quad \text { and } \quad A=A^{*}=\frac{\beta}{1-\alpha} .
$$

The values $B^{*}$ and $A^{*}$ are the so-called WALD approximations for the stopping boundaries.

A sufficient condition for an admissible test for the hypotheses (2) at size $(\alpha, \beta)$ is $B=\beta$ and $A=1 / \alpha$. Then we have $Q\left(\vec{\theta}_{0}\right) \geq 1-\alpha$ and $Q\left(\vec{\theta}_{1}\right) \leq \beta$.

\section{The ASN-function}

By means of the moment equation $E_{\vec{\theta}} Z_{N, \vec{\theta}_{0}, \vec{\theta}_{1}}=E_{\vec{\theta}} N$. $E_{\vec{\theta}} Z_{1, \vec{\theta}_{0}, \vec{\theta}_{1}}$ which holds for our tests if, e.g., $P_{\vec{\theta}}\left(L_{1, \vec{\theta}_{0}, \vec{\theta}_{1}}=\right.$ $1)<1$ we get in case of $E_{\vec{\theta}} Z_{1, \vec{\theta}_{0}, \vec{\theta}_{1}} \neq 0$ for the average sample number

$$
\begin{aligned}
& E_{\vec{\theta}} N=\left(E_{\vec{\theta}}\left(Z_{N, \vec{\theta}_{0}, \vec{\theta}_{1}} \mid H_{0} \text { is acc. }\right) Q(\vec{\theta})\right. \\
& +E_{\vec{\theta}}\left(Z_{N, \vec{\theta}_{0}, \vec{\theta}_{1}} \mid H_{1} \text { is acc. }\right)(1-Q(\vec{\theta})) / E_{\vec{\theta}} Z_{1, \vec{\theta}_{0}, \vec{\theta}_{1}} .
\end{aligned}
$$

If we again assume that condition (5) holds approximately we obtain the so-called WALD approximation $E_{\vec{\theta}}^{*} N$ for the average sample number $E_{\vec{\theta}} N$ :

$$
E_{\vec{\theta}} N \approx E_{\vec{\theta}}^{*} N=\frac{\ln B Q^{*}(\vec{\theta})+\ln A\left(1-Q^{*}(\vec{\theta})\right)}{E_{\vec{\theta}} Z_{1, \vec{\theta}_{0}, \vec{\theta}_{1}}} .
$$

In case of $E_{\vec{\theta}} Z_{1, \vec{\theta}_{0}, \vec{\theta}_{1}}=0$ we get by means of the moment equation $E_{\vec{\theta}} Z_{N, \vec{\theta}_{0}, \vec{\theta}_{1}}^{2}=E_{\vec{\theta}} N \cdot E_{\vec{\theta}} Z_{1, \vec{\theta}_{0}, \vec{\theta}_{1}}^{2}$ the approximation $E_{\vec{\theta}} N \approx E_{\vec{\theta}}^{*} N=-\ln B \ln A / E_{\vec{\theta}} Z_{1, \vec{\theta}_{0}, \vec{\theta}_{1}}^{2}$.

\section{E. Conjugated parameter pairs}

According to our definition of conjugated parameter pairs we have in the i.i.d. case the following criterion. It holds $\left(\vec{\theta}, \vec{\theta}^{\prime}\right) \stackrel{h}{\sim}\left(\vec{\theta}_{0}, \vec{\theta}_{1}\right)$ if to a given parameter vector $\vec{\theta} \in \Theta$ a real number $h \neq 0$ and a parameter vector $\vec{\theta}^{\prime} \in \Theta$, $\overrightarrow{\theta^{\prime}} \neq \vec{\theta}$ exist such that

$$
\frac{f_{\vec{\theta}^{\prime}}(x)}{f_{\vec{\theta}}(x)}=\left(\frac{f_{\vec{\theta}_{1}}(x)}{f_{\vec{\theta}_{0}}(x)}\right)^{h}
$$

holds for $x \in \mathcal{X}, \mathcal{X}$ the domain of $X_{1}$. Hence, a necessary existence condition for conjugated parameter pairs is, that function

$$
f_{\vec{\theta}^{\prime}}(x)=\left(\frac{f_{\vec{\theta}_{1}}(x)}{f_{\vec{\theta}_{0}}(x)}\right)^{h} f_{\vec{\theta}}(x), \quad x \in \mathcal{X},
$$

is a density function. Because of $f_{\vec{\theta}^{\prime}}(x) \geq 0$ for $x \in \mathcal{X}$ we can compute a value $h,-\infty<h<\infty$, such that

$$
\begin{aligned}
\varphi_{\vec{\theta}}(h)=\int_{-\infty}^{\infty} f_{\vec{\theta}^{\prime}}(x) d x=\int_{-\infty}^{\infty} & \left(\frac{f_{\vec{\theta}_{1}}(x)}{f_{\vec{\theta}_{0}}(x)}\right)^{h} f_{\vec{\theta}}(x) d x \\
& =E_{\vec{\theta}} \mathrm{e}^{h Z_{1, \vec{\theta}_{0}, \vec{\theta}_{1}}}=1 .
\end{aligned}
$$

The function $\varphi_{\vec{\theta}}(h)$ is as function of $h,-\infty<h<$ $\infty$, the moment-generating function of the random variable $Z_{1, \vec{\theta}_{0}, \vec{\theta}_{1}}=\ln L_{1, \vec{\theta}_{0}, \vec{\theta}_{1}}$. It holds $\varphi_{\vec{\theta}}(0)=1$, $\lim _{h \rightarrow \pm \infty} \varphi_{\vec{\theta}}(h)=\infty, \varphi_{\vec{\theta}}^{\prime}(0)=E_{\vec{\theta}} Z_{1, \vec{\theta}_{0}, \vec{\theta}_{1}}$ as well as $\varphi_{\vec{\theta}}^{\prime \prime}(h)=E_{\vec{\theta}}\left(Z_{1, \vec{\theta}_{0}, \vec{\theta}_{1}}^{2} \mathrm{e}^{\left.h Z_{1, \vec{\theta}_{0}, \vec{\theta}_{1}}\right)}>0\right.$. This means, that $\varphi_{\vec{\theta}}(h)$ is a convex function in $h$. Hence, we have in case of $E_{\vec{\theta}} Z_{1, \vec{\theta}_{0}, \vec{\theta}_{1}}<0$ and $E_{\vec{\theta}} Z_{1, \vec{\theta}_{0}, \vec{\theta}_{1}}>0$ beside the trivial solution $h=0$ of equation $\varphi_{\vec{\theta}}(h)=1$ always an unique solution $h>0$ and $h<0$, respectively.

If condition (5) holds approximately we have in case of $\left(\vec{\theta}, \vec{\theta}^{\prime}\right) \stackrel{h}{\sim}\left(\vec{\theta}_{0}, \vec{\theta}_{1}\right)$

$$
Q(\vec{\theta}) \approx Q_{\vec{\theta}}^{*}(\vec{\theta})=\frac{A^{h}-1}{A^{h}-B^{h}} .
$$

An explicit determination of parameter vector $\vec{\theta}^{\prime}$ is not necessary then. 
0.95

0.05

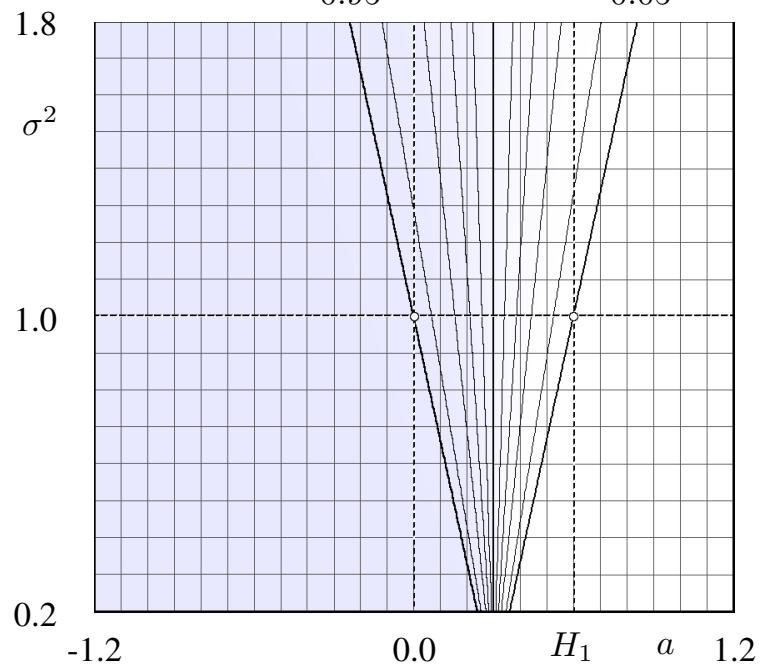

Fig. 1. Contour lines of OC-function $Q^{*}\left(a, \sigma^{2}\right)=q$ for $H_{0}: a_{0}=$ $0, \sigma_{0}^{2}=1, H_{1}: a_{1}=0.6, \sigma_{1}^{2}=1, q=0.05(0.05) 0.95$.

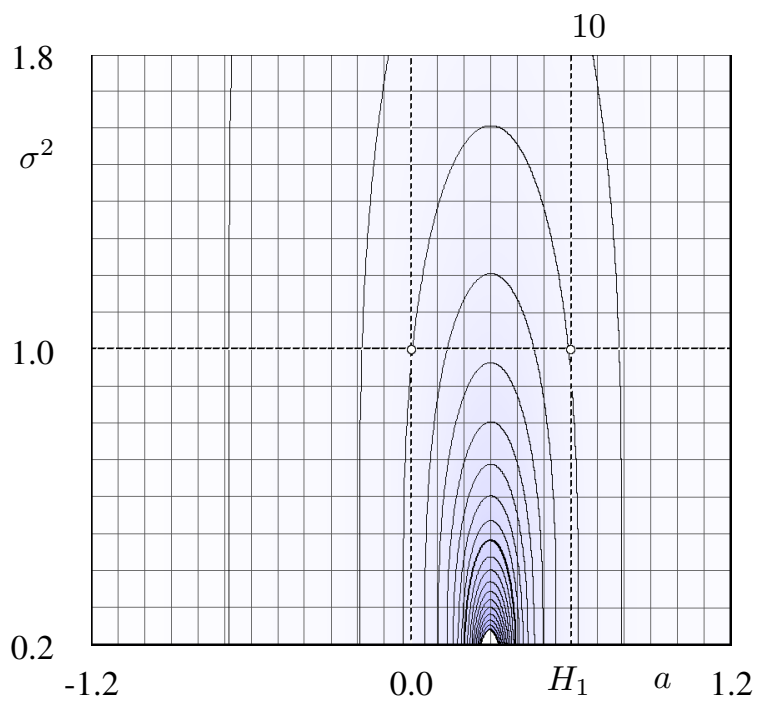

Fig. 2. Contour lines of ASN-function $E_{a, \sigma^{2}}^{*} N=e$ for $H_{0}: a_{0}=$ $0, \sigma_{0}^{2}=1, H_{1}: a_{1}=0.6, \sigma_{1}^{2}=1, e=0(5) 100$.

\section{EXAMPLE: NORMAL DISTRIBUTION}

Let $X_{1}, X_{2}, \ldots$ be independent $N\left(a, \sigma^{2}\right)$-distributed random variables. We consider an SPRT for discriminating between the simple hypotheses

$$
H_{0}: a=a_{0}, \sigma^{2}=\sigma_{0}^{2} \quad \text { and } \quad H_{1}: a=a_{1}, \sigma^{2}=\sigma_{1}^{2}
$$

where $a_{0} \neq a_{1}$ and/or $\sigma_{0}^{2} \neq \sigma_{1}^{2}$ holds. In case of $\sigma_{0}^{2}=\sigma_{1}^{2}$, $\sigma_{0}^{2}$ known, we have the usual one-dimensional test for the mean with hypotheses

$$
H_{0}: a=a_{0} \quad \text { and } \quad H_{1}: a=a_{1} .
$$

Analogously, we get in case of $a_{0}=a_{1}, a_{0}$ known, the corresponding one-dimensional test for the variance with hypotheses

$$
H_{0}: \sigma^{2}=\sigma_{0}^{2} \quad \text { and } \quad H_{1}: \sigma^{2}=\sigma_{1}^{2} .
$$

Let $f_{\vec{\theta}}(x)=f_{a, \sigma^{2}}(x)=\frac{1}{\sqrt{2 \pi}} \exp \left(-\frac{(x-a)^{2}}{2 \sigma^{2}}\right)$ be the density function of our observation variables $X_{1}, X_{2}, \ldots$.

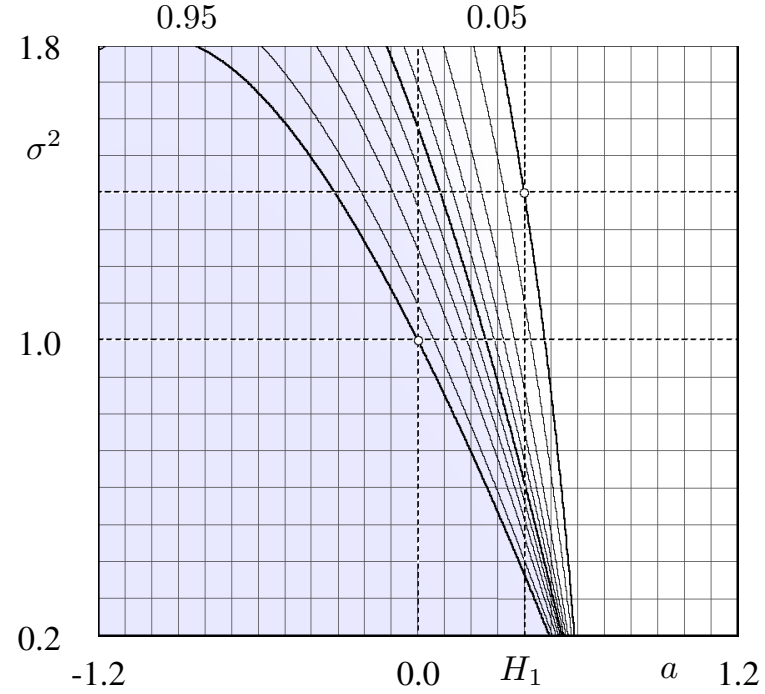

Fig. 3. Contour lines of OC-function $Q^{*}\left(a, \sigma^{2}\right)=q$ for $H_{0}: a_{0}=$ $0, \sigma_{0}^{2}=1, H_{1}: a_{1}=0.4, \sigma_{1}^{2}=1.4, q=0.05(0.05) 0.95$.

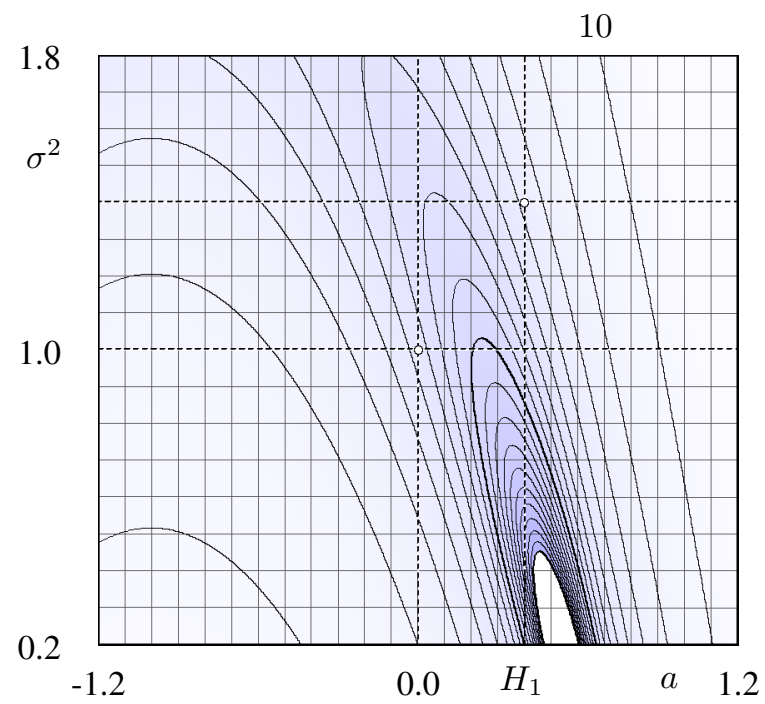

Fig. 4. Contour lines of ASN-function $E_{a, \sigma^{2}}^{*} N=e$ for $H_{0}: a_{0}=$ $0, \sigma_{0}^{2}=1, H_{1}: a_{1}=0.4, \sigma_{1}^{2}=1.4, e=0(5) 100$.

Then for the likelihood ratio for hypotheses (1) we obtain on stage $n, n=1,2, \ldots$,

$$
\begin{aligned}
L_{n, \vec{\theta}_{0}, \vec{\theta}_{1}} & =L_{n, a_{0}, \sigma_{0}^{2}, a_{1}, \sigma_{1}^{2}}=\prod_{i=1}^{n} \frac{f_{a_{1}, \sigma_{1}^{2}}\left(X_{i}\right)}{f_{a_{0}, \sigma_{0}^{2}}\left(X_{i}\right)} \\
& =\prod_{i=1}^{n} \exp \left(c_{0}+c_{1} X_{i}+c_{2} X_{i}^{2}\right),
\end{aligned}
$$

where

$$
c_{0}=\ln \frac{\sigma_{0}}{\sigma_{1}}+\frac{1}{2}\left(\frac{a_{0}^{2}}{\sigma_{0}^{2}}-\frac{a_{1}^{2}}{\sigma_{1}^{2}}\right), \quad c_{1}=\frac{a_{1}}{\sigma_{1}^{2}}-\frac{a_{0}}{\sigma_{0}^{2}}
$$

and

$$
c_{3}=\frac{1}{2}\left(\frac{1}{\sigma_{0}^{2}}-\frac{1}{\sigma_{1}^{2}}\right)
$$

holds.

The WALD approximations for the OC- and ASNfunction: Following the concept of conjugated parameter 


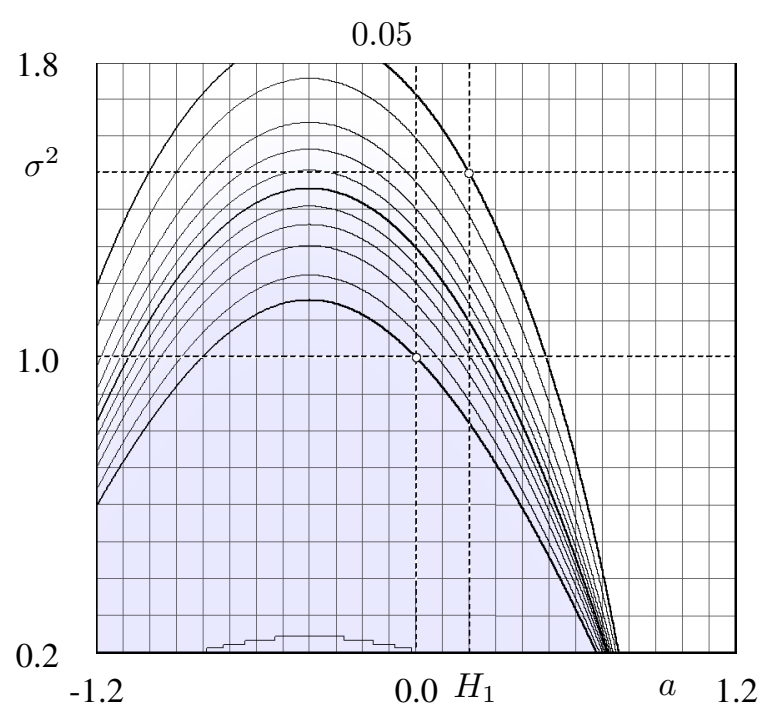

Fig. 5. Contour lines of OC-function $Q^{*}\left(a, \sigma^{2}\right)=q$ for $H_{0}: a_{0}=$ $0, \sigma_{0}^{2}=1, H_{1}: a_{1}=0.2, \sigma_{1}^{2}=1.5, q=0.05(0.05) 0.95$.

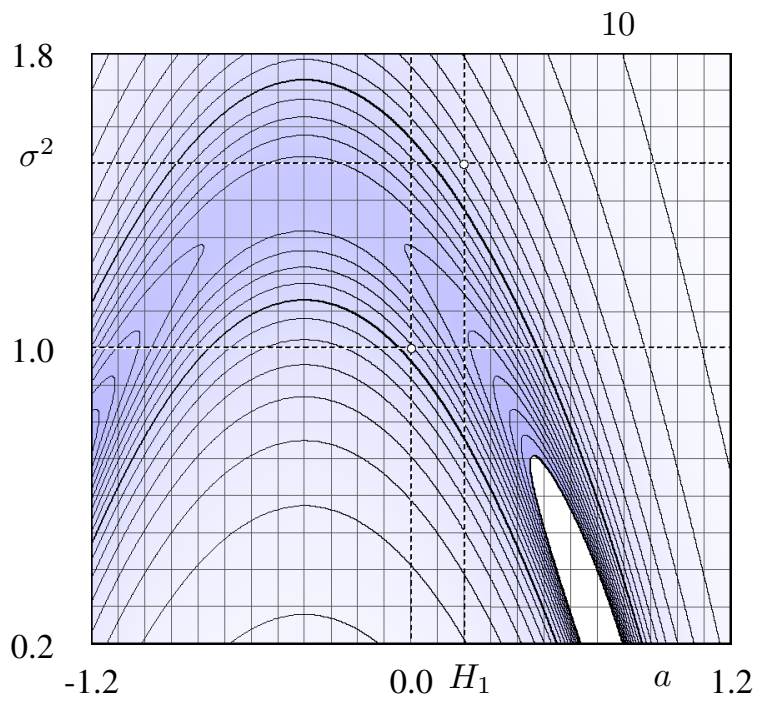

Fig. 6. Contour lines of ASN-function $E_{a, \sigma^{2}}^{*} N=e$ for $H_{0}: a_{0}=$ $0, \sigma_{0}^{2}=1, H_{1}: a_{1}=0.2, \sigma_{1}^{2}=1.5, e=0(5) 100$.

pairs we have to calculate to given parameter values $a$ and $\sigma^{2}$ conjugated parameter values $a^{\prime}$ and ${\sigma^{\prime}}^{2}$ such that

$$
L_{n, a, \sigma^{2}, a^{\prime}, \sigma^{\prime 2}}=L_{n, a_{0}, \sigma_{0}^{2}, a_{1}, \sigma_{1}^{2}}^{h}, \quad n=1,2, \ldots
$$

holds. Because of $X_{1}, X_{2}, \ldots$ are assumed to be i.i.d. random variables this condition is equivalent to

$$
L_{1, a, \sigma^{2}, a^{\prime}, \sigma^{\prime 2}}=L_{1, a_{0} \sigma_{0}^{2}, a_{1}, \sigma_{1}^{2}}^{h}
$$

Let $Z_{1, a, \sigma^{2}, a^{\prime}, \sigma^{\prime 2}}$ and $Z_{1, a_{0}, \sigma_{0}^{2}, a_{1}, \sigma_{1}^{2}}$ be the logarithms of $L_{1, a, \sigma^{2}, a^{\prime}, \sigma^{\prime 2}}$ and $L_{1, a_{0} \sigma_{0}^{2}, a_{1}, \sigma_{1}^{2}}$ then we have

$$
Z_{1, a_{0}, \sigma_{0}^{2}, a_{1}, \sigma_{1}^{2}}=c_{0}+c_{1} X_{1}+c_{2} X_{1}^{2}
$$

and

$$
Z_{1, a, \sigma^{2}, a^{\prime}, \sigma^{\prime 2}}=c_{0}^{\prime}+c_{1}^{\prime} X_{1}+c_{2}^{\prime} X_{1}^{2},
$$

where $c_{0}^{\prime}, c_{1}^{\prime}$ and $c_{2}^{\prime}$ are coefficients defined in accordance to (9) and (10) for the parameter set $a, \sigma^{2}, a^{\prime}$ and ${\sigma^{\prime}}^{2}$. Then (11) is further equivalent to

$$
Z_{1, a, \sigma^{2}, a^{\prime}, \sigma^{\prime 2}}=h Z_{1, a_{0}, \sigma_{0}^{2}, a_{1}, \sigma_{1}^{2}}
$$

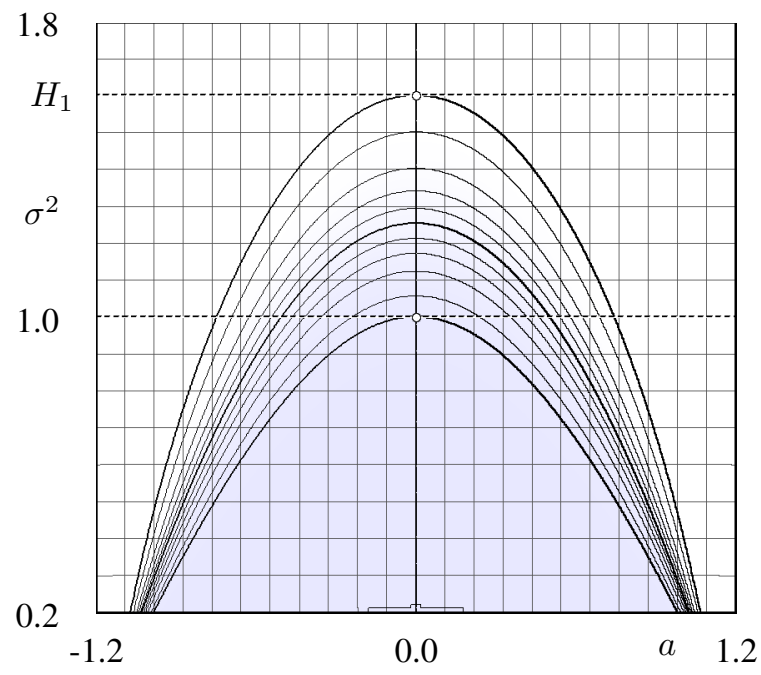

Fig. 7. Contour lines of OC-function $Q^{*}\left(a, \sigma^{2}\right)=q$ for $H_{0}: a_{0}=$ $0, \sigma_{0}^{2}=1, H_{1}: a_{1}=0, \sigma_{1}^{2}=1.6, q=0.05(0.05) 0.95$.

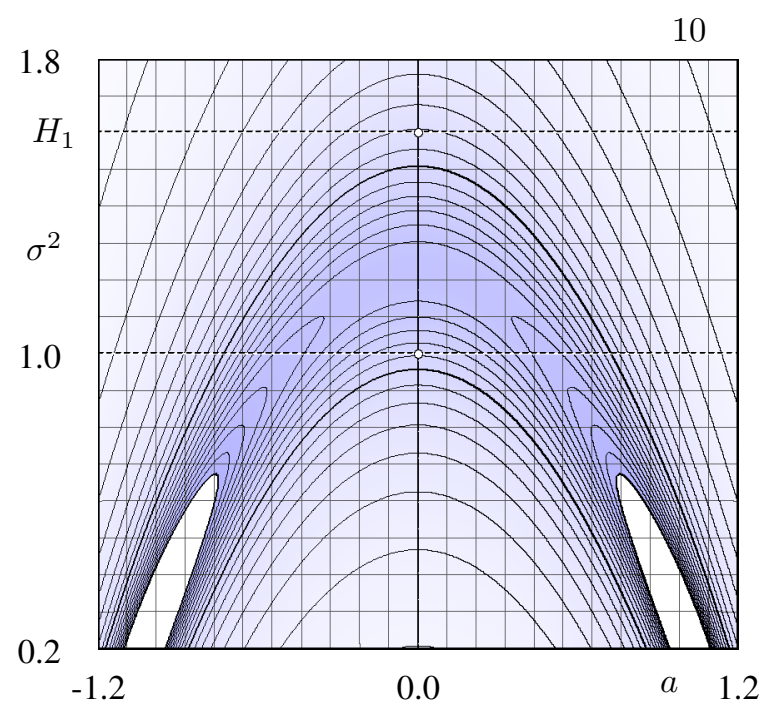

Fig. 8. Contour lines of ASN-function $E_{a, \sigma^{2}}^{*} N=e$ for $H_{0}: a_{0}=$ $0, \sigma_{0}^{2}=1, H_{1}: a_{1}=0, \sigma_{1}^{2}=1.6, e=0(5) 100$.

or

$$
c_{0}^{\prime}+c_{1}^{\prime} X_{1}+c_{2}^{\prime} X_{1}^{2}=h c_{0}+h c_{1} X_{1}+h c_{2} X_{1}^{2} .
$$

By means of comparison of coefficients this implies $\left(a, \sigma^{2}, a^{\prime},{\sigma^{\prime}}^{2}\right) \stackrel{h}{\sim}\left(a_{0}, \sigma_{0}^{2}, a_{1}, \sigma_{1}^{2}\right)$ if

$$
c_{0}^{\prime}=h c_{0}, \quad c_{1}^{\prime}=h c_{1} \quad \text { und } \quad c_{0}^{\prime}=h c_{2}
$$

holds. To given $a$ and $\sigma^{2}$ these three equations form a nonlinear system of equations with unknowns $h, a^{\prime}$ and ${\sigma^{\prime}}^{2}$, which can be solved by means of an appropriate iteration procedure. With respect to the WALD approximations of OC- and ASN-function, we are looking for, it is sufficient to compute then only the parameter value $h$.

Alternatively, the parameter value $h$ could be computed as follows. It holds $\left(a, \sigma^{2}, a^{\prime},{\sigma^{\prime}}^{2}\right) \stackrel{h}{\sim}\left(a_{0}, \sigma_{0}^{2}, a_{1}, \sigma_{1}^{2}\right)$ if

$$
f_{a^{\prime}, \sigma^{\prime 2}}(x)=\left(\frac{f_{a_{1}, \sigma_{1}^{2}}(x)}{f_{a_{0}, \sigma_{0}^{2}}(x)}\right)^{h} f_{a, \sigma^{2}}(x)
$$




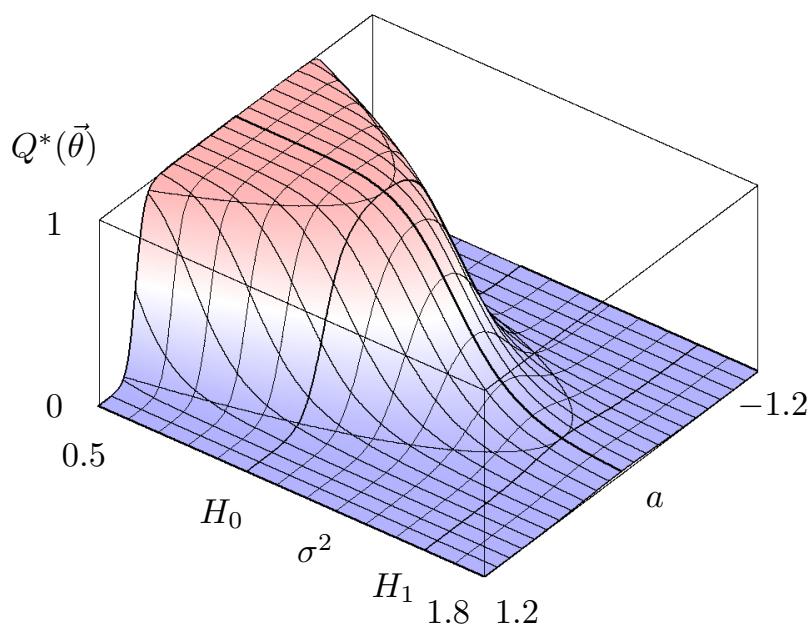

Fig. 9. OC-function $Q^{*}\left(a, \sigma^{2}\right)$ for $H_{0}: a_{0}=0, \sigma_{0}^{2}=1, H_{1}: a_{1}=$ $0, \sigma_{1}^{2}=1.6$.

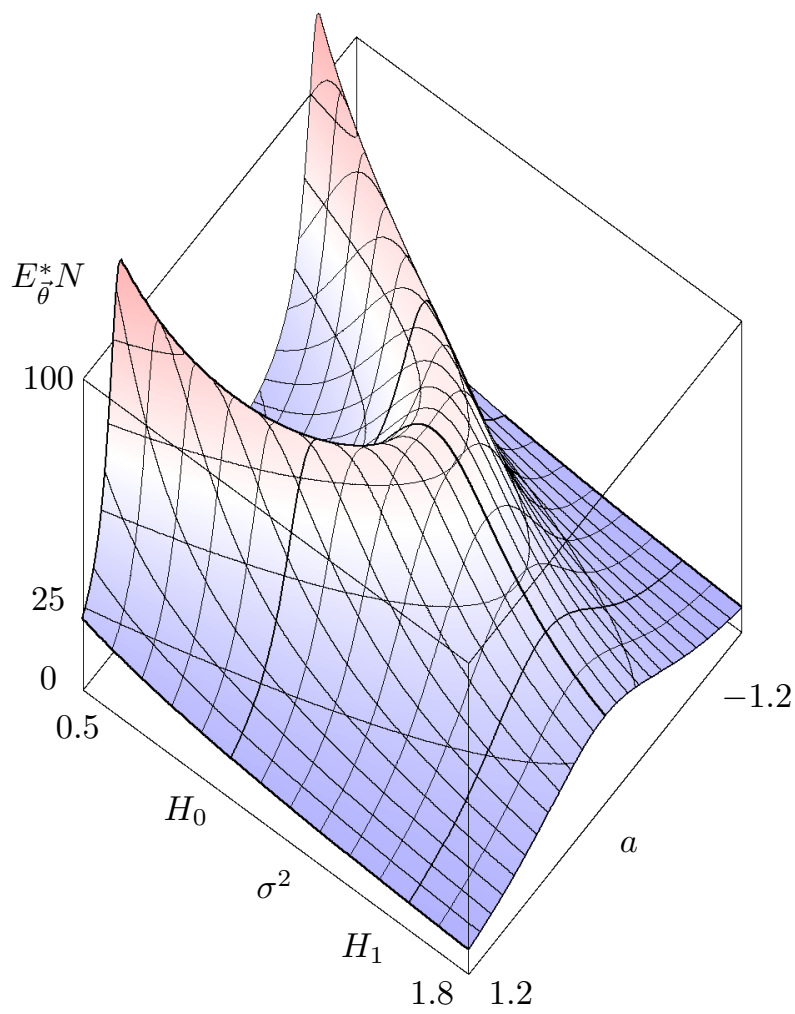

Fig. 10. ASN-function $E_{a, \sigma^{2}}^{*} N$ for $H_{0}: a_{0}=0, \sigma_{0}^{2}=1, H_{1}: a_{1}=$ $0, \sigma_{1}^{2}=1.6$.

is a density function. In this case we have

$$
\int_{-\infty}^{\infty} f_{a^{\prime}, \sigma^{\prime 2}}(x) d x=E_{a, \sigma^{2}} \mathrm{e}^{h Z_{1, a_{0}, \sigma_{0}^{2}, a_{1}, \sigma_{1}^{2}}}=1
$$

where $\varphi_{a, \sigma^{2}}(h)=E_{a, \sigma^{2}} \mathrm{e}^{h Z_{1, a_{0}, \sigma_{0}^{2}, a_{1}, \sigma_{1}^{2}}}$ for $-\infty<h<$ $\infty$ is the moment-generating function of $Z_{1, a_{0}, \sigma_{0}^{2}, a_{1}, \sigma_{1}^{2}}$. It can by shown, that in case of $P_{a, \sigma^{2}}\left(Z_{1, a_{0}, \sigma_{0}^{2}, a_{1}, \sigma_{1}^{2}}=\right.$ $0)<1$ this function is a convex function in $h,-\infty<$ $h<\infty$ with $\lim _{h \rightarrow \pm \infty} \varphi_{a, \sigma^{2}}(h)=\infty$. Because of $\varphi_{a, \sigma^{2}}^{\prime}(0)=E_{a, \sigma^{2}} Z_{1, a_{0}, \sigma_{0}^{2}, a_{1}, \sigma_{1}^{2}}$ and $\varphi_{a, \sigma^{2}}(0)=1$ equation $\varphi_{a, \sigma^{2}}(h)=1$ has a unique, non-zero solution $h$. This is again the parameter value $h$ needed in context of our principle of conjugated parameter pairs.

Some examples: Without loss of generality it can be assumed that $a_{0}=0$ and $\sigma_{0}^{2}=1$. Otherwise, this can be reached by transformation of $X_{1}, X_{2}, \ldots$ according $X_{i}^{\prime}=$ $\frac{X_{i}-a_{0}}{\sigma_{0}}, i=1,2, \ldots$ The Figures 1-10 demonstrate the behavior of OC- and ASN-function of SPRTs for

$$
H_{0}: a=0, \sigma^{2}=1 \quad \text { and } H_{1}: a=a_{1}, \sigma^{2}=\sigma_{1}^{2}
$$

depending on different alternative hypotheses to given error probabilities $\alpha=\beta=0.05$ of an error of first or second kind and stopping boundaries $B=B^{*}$ and $A=A^{*}$ according to (6). Interesting is the behavior of OC-function in case of $a_{0}=a_{1}$, see Fig. 7, which corresponds the one-dimensional test for the variance with known mean. For small or large values of parameter $a$ this test tends for $\sigma^{2}<\sigma_{0}^{2}$ again to the acceptance of hypothesis $H_{1}: \sigma^{2}=\sigma_{0}^{2}$. Moreover, the examples considered here show how in certain cases tests for composite hypotheses $H_{0}:\left(a, \sigma^{2}\right) \in G_{0}$ against $H_{1}:\left(a, \sigma^{2}\right) \in G_{1}$, $G_{0} \cap G_{1}=\emptyset$, can be reduced to tests for proper chosen simple hypotheses.

\section{REFERENCES}

[1] V.I. Denisov, K.-H. Eger, B.Yu. Lemesko, and E.B. Tsoy, Design of experiments and statistical analysis for grouped observations. Novosibirsk, NSTU Publishing House, 2004.

[2] K.-H. Eger, "Likelihood ratio tests for grouped observations", Chemnitz University of Technology, Faculty of Mathematics, Preprint 2003-10, 2003.

[3] K.-H. Eger, Sequential tests. Teubner, Leipzig, 1985.

[4] K.-H. Eger, E.B. Tsoy, "Sequential probability ratio tests based on grouped observations", Proceedings of the Second International Forum on Strategic Technology - IFOST 2007, pp. 284-287, Ulaanbaatar, Mongolia, 2007.

[5] B.K. Ghosh, Sequential Tests of Statistical Hypotheses. AddisonWessley, Reading, Mass, 1970.

[6] B.K. Ghosh, P.K. Sen (editors), Handbook of Sequential Analysis. Marcell Dekker, Inc., New York, 1991.

[7] A. Wald, Sequential Analysis. Wiley, New York, 1947.
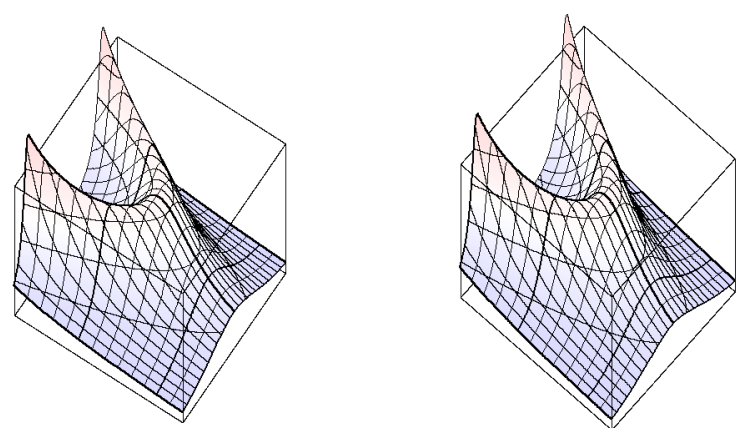

Fig. 11. Three dimensional version of Fig. 10. 